\title{
IMPACT OF MIGRATION BACKGROUND ON HEALTH OF CHILDREN AND ADOLESCENTS IN AUSTRIA RESULTS OF THE AUSTRIAN HEALTH SURVEY 2014
}

\author{
Anja Waxenegger, Hannes Mayerl, Erwin Stolz, Éva Rásky, Wolfgang Freidl \\ Institute of Social Medicine and Epidemiology, Medical University of Graz, Graz, Austria
}

\section{SUMMARY}

Objective: The present study examines the question as to whether the wellbeing of children and adolescents in Austria and the use of vaccination are influenced by migration background.

Methods: The data was extracted from the Austrian Health Survey 2014 (ATHIS 2014). It contains health-related information of 5,277 children and adolescents aged 0 to 17 years. The analysis was based on logistic regression models.

Results: To stem from a migration background had no influence on the assessment of health or the assessment of complaints. Regarding vaccination, the results showed that the children of study participants born in non-EU foreign countries had twice the chance of being vaccinated as the children of Austria-born parents. No difference existed between the children of Austria-born parents and the children of parents born in non-EU countries.

Conclusions: The analysis suggests that children and adolescents with a migration background in Austria are not worse off in terms of their physical wellbeing and vaccination status than children without a migration background. Some thought should, however, be given to the fact that the notion of migration background refers to a heterogenic population. To analyse the risks and chances of children and adolescents from different migrant backgrounds, a more differentiated survey of their migrant background and social situation and a more differentiated survey of health parameters will be required.

Key words: children's health, adolescents' health, migration background, ATHIS 2014, vaccination

Address for correspondence: W. Freidl, Institute of Social Medicine and Epidemiology, Medical University of Graz, Universitätsstrasse 6/l, 8010 Graz, Austria. E-mail: wolfgang.freidl@medunigraz.at

https://doi.org/10.21101/cejph.a4917

\section{INTRODUCTION}

Health-related life course research has shown childhood and youth to play an important role for the further health biography. Surveys have revealed children and adolescents from socioeconomically less well-off families to be more frequently exposed to health burdens than children and youth from wealthier families (1, 2 ). The health situation of children and adolescents with a migration background has scarcely been discussed to date. Nonetheless, surveys on this issue indicate that children and adolescents with a migration background tend to have a poorer health status than those without migration background. The picture becomes more complex when it comes to medical care. In certain areas, for example in prevention, we found that children of migrants are harder to reach by the healthcare system (3-5).

Generally speaking, the status of research on the health of children and adolescents in Austria is moderately low. So far, representative survey data have only been gathered for groups of pupils aged 11,13,15, or 17 as part of the Health Behaviour in School-Aged Children Survey (HBSC) (3). Unlike in Germany, where such data are sampled by the Children and Youth Health Survey (KiGGS), population-wide representative health data for the 0 to 17 -year-olds in Austria were mostly inexistent (4). This gap has now been filled partly by the Austrian Health Survey 2014 (ATHIS). Since the current dataset also includes information about migration background, Austria now for the first time avails of representative data on the health of 0 to 17 -year-old children and adolescents with a migration background $(6,7)$.

Given the current influx of refugees into Austria and the great significance of childhood and youth for the later health status of a person, information about the health of children and adolescents with a migration background and their use of preventive measures are of particular relevance. The present study explored the question whether the fact of being from a migration background affected the health status and use of vaccination for children and adolescents in Austria.

\section{MATERIALS AND METHODS}

\section{Sample}

The analysis is based on data from the current Austrian Health Interview Survey 2014 (ATHIS 2014). The Austria-wide survey 
based on the European Health Survey (EHIS) has been adopted by an expert panel. It was conducted from October 2013 to June 2015 by the Statistics Austria on behalf of the Austrian Ministry of Health. The random sample was drawn from the central population register and stratified by geographic region. The gross sample size consisted of 38,768 people aged 15 years and older. Data was collected using computer assisted telephone interviewing (CATI) and a written questionnaire, which has to be completed by the respective recipients. The net sample size comprised 15,771 participants (response rate 40.7\%). The interviewees are representative of the Austrian population.

ATHIS 2014 included health-related data for 5,277 children and adolescents aged 0 to 17 years (2,730 boys and 2,547 girls). The age distribution was as follows: 0-2 years $(n=829), 3-6$ years $(n=1,087), 7-10$ years $(n=1,164), 11-13$ years $(n=921)$, and $14-17$ years $(n=1,276)$. Among migrants, 309 participants (62.8\%) came to Austria before 2000, 122 participants (19.6\%) came in the years $2001-2005,84$ participants $(13.5 \%)$ in the years 2006-2010, and 25 participants (4.0\%) have immigrated to Austria after 2010. Country of origin of all participants can be found in Table 1. All data were based on the statements of parents and referred to children living in the same household (7).

\section{Dependent Variables}

ATHIS 2014 assessed the health of these children and adolescents with a number of different items. These items included, among other things, a question regarding the subjective state of health. Parents answered the question "How would you describe your child's general state of health?" using a 5-point rating scale, ranging from $1=$ very good to 5 = very poor. Research has generally accepted the subjective assessment of a person's health status as an adequate indicator for health and a sound predictor for the use of healthcare system (4), and this item was also used for health assessment in the present analysis. The distribution of this variable led us to dichotomize this item. Answer categories 1

Table 1. Country of origin of all participants $(N=5,277)$

\begin{tabular}{|l|c|c|}
\hline & $\mathbf{n}$ & $\%$ \\
\hline Austria & 4,645 & 88.02 \\
\hline Germany & 172 & 3.26 \\
\hline EU (excl. Austria and Germany) & 113 & 2.14 \\
\hline Bosnia and Herzegovina & 80 & 1.52 \\
\hline Bulgaria, Romania & 51 & 1.10 \\
\hline Turkey & 42 & 0.80 \\
\hline Serbia & 39 & 0.74 \\
\hline Kosovo, Macedonia, Montenegro & 30 & 0.57 \\
\hline Asia & 27 & 0.51 \\
\hline Switzerland & 21 & 0.40 \\
\hline Africa & 17 & 0.32 \\
\hline Other European countries & 15 & 0.28 \\
\hline Croatia & 10 & 0.19 \\
\hline Latin Amerika & 9 & 0.17 \\
\hline North Amerika & 6 & 0.11 \\
\hline
\end{tabular}

$=$ very good and $2=$ good were grouped into one category: good to very good state of health, and answer categories $3=$ mediumranged, $4=$ poor and $5=$ very poor were grouped into category: medium-ranged to very poor state of health.

In addition, participants were questioned about health-related complaints. The variable (at least one health-related complaint exists vs. no health-related complaints exist) was derived from the following statements: "My child is restless, hyperactive, finds it hard to sit still over a longer period." "My child often complains about headache, bellyache, or nausea.", and the question "Does your child suffer from sleeplessness?".

The natural candidate for implementing preventive measures in the current ATHIS dataset was the use of vaccination ("Do you make use of the free vaccination scheme for your child?" - "yes vs. no"). Only the data of children and adolescents from the age of 3 onwards were used for analysis, since a number of vaccines are only recommended from a certain age onwards (e.g. vaccines against rubeola/mumps/rubella, for which the 1st partial vaccination is only recommended from month 11 onwards and the 2nd partial vaccination 4 weeks after the first one at the earliest).

\section{Independent Variables}

The Statistics Austria combined the dataset from ATHIS 2014 with the current micro census data. This provided additional information about the migration background of children and adolescents. The relevant indicator was the native country of the questioned parent, with answer categories EU foreign country, non-EU foreign country, and Austria (7).

To allow for the fact that the family background influences the health of children and adolescents by a number of factors, we included other potential influencing factors for analysis, in addition to migration background. These referred to the family socioeconomic situation, number of siblings, parents' state of health, and family structure $(2,8)$. The socioeconomic situation was operationalized using the variable household income (income of all household members including social transfer payments such as family allowance etc.). The indicated figures were divided by the weighted number of household members and subsequently divided into five equally sized income groups. The number of siblings was assessed asking "How many children under 18 live in your household?"; the health status of the questioned parent asking "Have you got a chronic disease or a chronic health problem?"; and the family structure was assessed via the variable marital status and the categories cohabiting with a partner vs. not cohabiting with a partner. In order to account for the health status of different age groups, we defined the age of children and adolescents as a categorical variable. For the categorisation of age groups, the present analysis was based on procedures of the Robert Koch Institute and Statistics Austria $(4,7)$. Additional variables were the sex of the interviewees and the sex of the child/adolescent. Moreover, the results of the analysis were adjusted for region-specific influences, by integrating the variable degree of urbanisation with the categories low, medium, and high urbanization.

\section{Statistical Analysis}

Logistic regression analysis was used to determine a possible link between migration background and state of health and be- 
tween migration background and use of the vaccination scheme. To achieve this, we first exclusively considered the influence of migration background on the dependent variables (general health status, health complaints, use of vaccination) (Step 1). Subsequently, all potential influencing factors were considered (Step 2). All computations were done using SPSS (Version 23).

\section{RESULTS}

\section{Health Status}

Bivariate analysis has shown no significant association between migration background and the general state of health, or between migration background and health complaints. This result was reproduced in multivariate analysis. The proportion of children and adolescents whose general health status was rated by their own parents as very good or good was $96.8 \%$ for children and adolescents without a migration background, as opposed to $96.2 \%$ for those with a migration background from "EU foreign countries", and 95.1\% for children and adolescents from a "non-EU foreign" background. At least one complaint was found for $35.4 \%$ of children and adolescents without a migration background, for children and adolescents from an "EU foreign" migrant background this percentage was $33.5 \%$, and for children and adolescents from a "non-EU foreign" background it was $35.3 \%$.

The multivariate computation found age-specific differences in the assessment of general health status, regardless of the migration background. The three younger age groups ( $0-10$ years) tended to be rated better than the 14 to 17 -year-olds. The assessment of 11 to 13 -year-olds, however, showed no difference with regard to the reference group. The analysis also revealed that children and adolescents with two or more siblings, as opposed to singletons, were more likely to be assessed by their parents as being in very good or in good health. Other significant influencing factors included the health status and the marital status of the questioned parent. The presence of a chronic health condition in a parent lowered the chance for children and adolescents to be ascribed a very good or good health status. The same was true for children and adolescents whose fathers and/or mothers were not living with a partner. Compared to children and adolescents whose parents were living in a partnership, these children and adolescents had a clearly lower chance to be ascribed a good or very good health status. Thus the chance of being assessed as being in good health was almost twice as high for children and adolescents whose parents lived in a partnership.

As opposed to the 14 to 17 -year-olds, children and adolescents aged 3 to 13 showed a higher risk of having at least one health problem. Babies and toddlers (0 to 2 years of age) showed no significant difference with regard to the 14 to 17 -year-olds in this respect. That children and adolescents with more than one sibling tended to receive a better health ranking also applied to health complaints. The lower risk, compared to those who grew up as an only child, likewise applied to children and adolescents with only one sibling in this respect. Another significant factor was the socioeconomic situation of parents. Compared to children and adolescents who grew up in families of the lowest income group, children and adolescents from the three higher income groups had a lower risk for potential health complaints. Families from the lower two income classes showed no difference regarding their risk for health complaints. That children and adolescents with a chronically ill parent have a higher risk of health burdens was also confirmed when asking for complaints, albeit in a lower intensity. Compared to children whose parents lived alone, children and adolescents of parents living in a partnership had a lower risk also in this respect. In addition, our study revealed that the risk of health complaints was slightly higher in male children and adolescents than in females. A significant link was found between the sex of the interviewed parent and the given health assessment. For example, mothers tended to give somewhat poorer assessments of their children regarding health complaints than fathers.

\section{Use of Vaccination}

As shown in Table 2, the use of vaccination scheme was influenced by migration status. The chance of being vaccinated was twice as high in children and adolescents with a parent born in a "non-EU foreign country" than for children and adolescents whose parent was born in Austria. This likelihood ratio remained in the multivariate analysis that accounted for the influence of several variables. No difference regarding the use of vaccination scheme for their children was found between "EU foreigners" and born Austrians, neither in the bivariate nor in the multivariate analysis. The percentage of unvaccinated children and adolescents was $5.1 \%$ among children and adolescents with a "non-EU" migrant background, $8.1 \%$ among children and adolescents with an "EU migrant" background, and 9.8\% among children and adolescents without a migration background.

In addition, we showed that the chance of being vaccinated was higher for children and adolescents from families with a medium income than for children and adolescents from the lowest income group. Compared to the lowest income group the two highest income groups, however, showed no difference regarding the use of vaccination scheme.

\section{DISCUSSION}

Our analysis revealed no link between the factor "migration background" and the health of children and adolescents. This contradicts the results of other analyses and surveys, which found a tendency toward a poorer assessment of subjective health among children and adolescents with a migration background (3-5). This discrepancy may, however, also be due to a difference in operationalizing the notion of "migration background". For instance, the HBSC and KiGGS surveys distinguished between a bilateral migration background (child/adolescent born abroad and at least one parent born abroad/of foreign nationality or both parents born abroad/of foreign nationality) and a unilateral migration background (one parent born abroad/of foreign nationality). The association between the migration background and the health of children and adolescents, which has been found in the HBSC survey 2014 and the KiGGS survey 2003-2006, is solely based on data on children and adolescents with bilateral migration background $(3,4)$. ATHIS 2014 defined the migration background of the child/adolescent based on the state of birth of one parent. As a consequence, the results are only partly comparable. 
Table 2. Influence of migration background and other family factors on the general health status, health complaints, and use of the vaccination scheme of children and adolescents $(N=5,277)$

\begin{tabular}{|c|c|c|c|c|c|c|c|c|}
\hline \multirow{5}{*}{ Step 1} & \multirow{4}{*}{$\begin{array}{l}\text { Migration background } \\
\text { EU foreign country }\end{array}$} & \multirow{4}{*}{$\begin{array}{c}\text { n } \\
\text { No migrant background: 4,645 } \\
346\end{array}$} & \multicolumn{2}{|c|}{$\begin{array}{l}\text { General health status } \\
\text { very good or good }\end{array}$} & \multicolumn{2}{|c|}{$\begin{array}{l}\text { At least one health } \\
\text { complaint }\end{array}$} & \multicolumn{2}{|c|}{$\begin{array}{l}\text { Use of vaccination } \\
\text { scheme: yes }\end{array}$} \\
\hline & & & OR & $95 \% \mathrm{Cl}$ & OR & $95 \% \mathrm{Cl}$ & OR & $95 \% \mathrm{Cl}$ \\
\hline & & & \multicolumn{2}{|c|}{ Reference } & \multicolumn{2}{|c|}{ Reference } & \multicolumn{2}{|c|}{ Reference } \\
\hline & & & 0.85 & $0.48-1.51$ & 0.92 & $0.73-1.16$ & 1.23 & $0.79-1.93$ \\
\hline & Non-EU foreign country & 286 & 0.64 & $0.37-1.13$ & 0.99 & $0.77-1.28$ & $2.04^{*}$ & $1.13-3.68$ \\
\hline \multirow{24}{*}{ Step 2} & Migration background & No migrant background: 4,645 & \multicolumn{2}{|c|}{ Reference } & \multicolumn{2}{|c|}{ Reference } & \multicolumn{2}{|c|}{ Reference } \\
\hline & EU foreign country & 346 & 0.80 & $0.44-1.44$ & 0.94 & $0.74-1.19$ & 1.26 & $0.80-1.98$ \\
\hline & Non-EU foreign country & 286 & 0.65 & $0.36-1.17$ & 1.01 & $0.77-1.31$ & $1.98^{*}$ & $1.08-3.62$ \\
\hline & Age of child & $14-17$ years: 1,276 & \multicolumn{2}{|c|}{ Reference } & \multicolumn{2}{|c|}{ Reference } & \multicolumn{2}{|c|}{ Reference } \\
\hline & Up to 2 years & 829 & $2.26^{* *}$ & $1.30-3.91$ & 1.19 & $0.98-1.44$ & - & - \\
\hline & 3 to 6 years & 1,087 & $1.84^{* *}$ & $1.16-2.92$ & $1.58^{* * *}$ & $1.32-1.89$ & 0.80 & $0.60-1.05$ \\
\hline & 7 to 10 years & 1,164 & $2.37^{* * *}$ & $1.46-3.83$ & $1.56^{* \star *}$ & $1.31-1.86$ & 0.97 & $0.73-1.29$ \\
\hline & 11 to 13 years & 921 & 1.21 & $0.80-1.84$ & $1.41^{* * *}$ & $1.18-1.69$ & 1.15 & $0.84-1.56$ \\
\hline & Sex of child & Girls: 2,547 & \multicolumn{2}{|c|}{ Reference } & \multicolumn{2}{|c|}{ Reference } & \multicolumn{2}{|c|}{ Reference } \\
\hline & Boys & 2,730 & 0.92 & $0.68-1.25$ & $1.13^{*}$ & $1.00-1.26$ & 1.05 & $0.86-1.28$ \\
\hline & Household income & $\begin{array}{l}\text { under 1st quintile: } 854 \\
\text { (lowest income group) }\end{array}$ & \multicolumn{2}{|c|}{ Reference } & \multicolumn{2}{|c|}{ Reference } & \multicolumn{2}{|c|}{ Reference } \\
\hline & 1st-2nd quintile & 1,436 & 1.01 & $0.65-1.58$ & 0.95 & $0.79-1.13$ & $1.44^{*}$ & $1.07-1.95$ \\
\hline & 2nd-3rd quintile & 1,147 & 1.08 & $0.66-1.75$ & $0.80^{*}$ & $0.66-0.97$ & $1.59^{* *}$ & $1.15-2.21$ \\
\hline & 3rd-4th quintile & 1,223 & 1.14 & $0.70-1.86$ & $0.77^{\star *}$ & $0.63-0.93$ & 1.34 & $0.97-1.84$ \\
\hline & $\begin{array}{l}\text { 4th-5th quintile (highest } \\
\text { income group) }\end{array}$ & 617 & 1.61 & $0.82-3.17$ & $0.77^{*}$ & $0.61-0.97$ & 1.19 & $0.81-1.74$ \\
\hline & Children in household & 1 child: 1,562 & \multicolumn{2}{|c|}{ Reference } & \multicolumn{2}{|c|}{ Reference } & \multicolumn{2}{|c|}{ Reference } \\
\hline & 2 children & 2,535 & 1.06 & $0.76-1.50$ & $0.81^{* *}$ & $0.70-0.92$ & 0.98 & $0.76-1.27$ \\
\hline & 3 children or more & 1,180 & $1.78^{*}$ & $1.08-2.93$ & $0.53^{* * *}$ & $0.44-0.63$ & 0.81 & $0.60-1.09$ \\
\hline & Health condition of parent & Chronic condition: 1,368 & \multicolumn{2}{|c|}{ Reference } & \multicolumn{2}{|c|}{ Reference } & \multicolumn{2}{|c|}{ Reference } \\
\hline & No chronic condition & 3,909 & $1.79^{* * *}$ & $1.31-2.46$ & $0.74^{* * *}$ & $0.65-0.85$ & 1.00 & $0.79-1.26$ \\
\hline & Marital status of parent & Living alone: 641 & \multicolumn{2}{|c|}{ Reference } & \multicolumn{2}{|c|}{ Reference } & & ence \\
\hline & Living with partner & 4,636 & $1.93^{\star *}$ & $1.31-2.85$ & $0.78^{* *}$ & $0.65-0.93$ & 1.03 & $0.75-1.42$ \\
\hline & Sex of parent & Male: 2,016 & & ence & & ence & & ence \\
\hline & Female & 3,261 & 0.81 & $0.57-1.14$ & $1.17^{*}$ & $1.03-1.32$ & 0.97 & $0.78-1.20$ \\
\hline
\end{tabular}

OR - odds ratio, $\mathrm{Cl}$ - confidence interval, ${ }^{*} \mathrm{p}=0.01-0.05,{ }^{* *} \mathrm{p}=0.001-0.009,{ }^{* * *} \mathrm{p}<0.001$, Step 2 : analyses were adjusted for region-specific influences by integrating the variable "degree of urbanisation" with the categories low, medium, and high urbanization

As for vaccination, no difference was found between individuals born in "EU foreign countries" and those born in Austria. The possibility that parents use the Austrian vaccination scheme for their children was clearly higher in those born in a "non-EU foreign country" than in parents of Austrian origin. Respondents born in "non-EU foreign countries" stated more frequently than Austrian-born respondents that they use the vaccination scheme for their children, regardless of the income category. One reason for this might be that parents born in "non-EU foreign countries" were less sceptical about vaccination than parents from Austria or "EU foreigners". However, the conclusion that children and adolescents with a migration background are well looked after in Austria, would be premature, as shown by the results of the KiGGS survey in Germany, where the initial outcome - showing no influence of the migration background on the vaccination of children or youth in respect of certain diseases - was not confirmed in a more detailed analysis. When splitting up children and adolescents with a migration background into those born in Germany and those having immigrated after their birth, it was found that the vaccination rate among children and adolescents who immigrated after their birth was not only much lower than in those without a migration background, but also much lower than the vaccination rate among children and adolescents with a migration background born in Germany $(4,9)$. A categorisation 
of the ATHIS data by the state of birth of children and adolescents was however impossible.

Another central outcome of our study was that the health of children and adolescents is influenced by family factors. Of particular importance in this respect seem the parents' health status and marital status. Several analyses have come to the conclusion that a low social status, from childhood or youth, increases the risk of health complaints $(1,2)$. The results of the present study point in the same direction.

The health of children and adolescents in ATHIS 2014 was assessed subjectively by their parents. A bias, e.g. due to erroneous assessment or socially desired answering, may thus not be excluded. However, Case and Paxon found in their comparison of parental information about the health of their children with medical diagnoses that the information parents provide about their children's health status can be regarded as valid (10). A potential limitation of our study is that, due to available data, no differential inferences between unilateral or bilateral migration background can be drawn. A further limitation concerns a potential bias due to the sampling strategy. The ATHIS survey is restricted to migrants who were able to master the interview/questionnaire in German language. The participating migrants might thus represent a rather selective sample, and the outcome measures might overestimate the health status of migrants and their children living in Austria. Moreover, differences in responding due to varying cultural backgrounds may hamper comparability between answers from Austrians and those from non-EU migrants to an unknowable degree. Hence, further studies based on more detailed data about the migration background of children and adolescents are required to substantiate our results.

\section{CONCLUSIONS}

The results of the current health survey revealed that children and adolescents with a migration background are not worse off in Austria regarding their health and vaccination status than children and adolescents without a migration background. This outcome should, however, not belie the existence of healthvulnerable groups among the children and adolescents of the migrant population now in Austria. More differentiated surveys will be required in order to make empirically sound statements regarding the health risks and chances and about the preventive behaviour and use of health services by children and adolescents from various migrant populations. Central aspects of children's and adolescents' health to be surveyed in future studies should include a more differentiated surveying of the migration background (e.g. unilateral or bilateral migration background), risk factors (such as body mass index, exercise, smoking), health status (e.g. assessment of motor function, linguistic development, seeing, hearing), complaints (e.g. headache, abdominal pain), use of preventive check-ups (e.g. children's and youth medicine, dental medicine), and chronic illnesses.
In conclusion, we may say that the results of the present study strengthen the hypothesis that the health of children and adolescents is linked to their family environment. This again underlines the high significance of measures to be taken for the support of families as part of health promotion and prevention programmes.

\section{Acknowledgements}

The authors would like to thank the Statistics Austria for providing data from the current Austrian Health Interview Survey 2014 (ATHIS 2014).

\section{Conflict of Interests}

None declared

\section{REFERENCES}

1. Dragano N, Siegrist J. A life course perspective on health inequalities: research concepts and results. In: Richter M, Hurrelmann K, editors. Health inequalities. Basics, problems, prospects. Wiesbaden: VS Verlag für Sozialwissenschaften; 2006. p. 171-84. (In German.)

2. Lampert T, Richter M. Health inequalities among children and adolescents. In: Richter M, Hurrelmann K, editors. Health inequalities. Basics, problems, prospects. Wiesbaden: VS Verlag für Sozialwissenschaften; 2006. p. 199-220. (In German.)

3. Federal Ministry of Health, editor. Health and health behaviour in schoolaged children. Results of the WHO-HBSC-Survey 2014. Vienna: Federal Ministry of Health; 2015. (In German.)

4. Robert Koch Institute, editor. Health Interview and Examination Survey for Children and Adolescents (KiGGS) 2003-2006: children and adolescents with migration background in Germany. Berlin: Robert Koch Institute; 2008. (In German.)

5. Ziol-Guest KM, Kalil A. Health and medical care among the children of immigrants. Child Dev. 2012 Sept-Oct;83(5):1494-500.

6. Czypionka T, Röhrling G. Analysis of the Austrian health-system due to the health of children and adolescents. In: Österreichische Liga für Kinder- und Jugendgesundheit, editor. Report on the health situation of children and adolescents in Austria. Vienna: Österreichische Liga für Kinder- und Jugendgesundheit; 2011. p. 25-8. (In German.)

7. Statistik Austria, editor. Austrian Health Interview Survey 2014. Results of the Austrian Health Interview Survey (ATHIS) and methodological documentation. Vienna: Statistik Austria; 2015. (In German.)

8. Rattay P, Lampert T, Neuhauser H, Ellert U. Significance of family life for the health of children and adolescents - Results of the German Health Interview and Examination Survey for Children and Adolescents (KiGGS). Z Erziehwiss. 2012 Mar;15(1):145-70. (In German.)

9. Poethko-Müller C, Ellert U, Kuhnert R, Neuhauser H, Schlaud M, Schenk L. Vaccination coverage against measles in German-born and foreignborn children and identification of unvaccinated subgroups in Germany. Vaccine. 2009 Apr 28;27(19):2563-9.

10. Case A, Paxson C. Parental behavior and child health. Health Aff (Millwood). 2002 Mar-Apr;21(2):164-78.

Received September 5, 2016 Accepted in revised form April 13, 2018 